\title{
Ecological boundaries: a derivative of ecological entities
}

\author{
J. Kolasa \\ Department of Biology, McMaster University, Hamilton, Ontario, L8S 4K1, Canada \\ Correspondence to: J. Kolasa (kolasa@mcmaster.ca)
}

Received: 4 March 2014 - Revised: 20 May 2014 - Accepted: 6 June 2014 - Published: 22 July 2014

\begin{abstract}
Defining ecological boundary as an outer envelope of an ecological entity such as an individual, colony, population, community, an ecosystem, or any other discernible unit provides methodological benefits and should thus enhance existing perspectives and research protocols. I argue that, because boundaries are features of entities, the first step in investigation of boundary structure and properties should involve identification of the entity the presumed boundary of interest belongs to. I use a general perspective where ecological systems are parts of a larger system and themselves are made of subsystems (or entities). Such a general hierarchy of ecological objects offers guidance as to how boundaries can be found for specific systems, and how their investigations might lead to reliable and generalizable insights. In particular, it may help in (a) categorizing types of boundaries based on mechanisms leading to formation of entities; (b) deciding what is and what is not a boundary by clarifying the nature of discontinuities seen in nature (e.g., sharp habitat transitions or weak separation of entities); (c) assisting in selecting fruitful resolution at which boundaries are examined; (d) approaching boundaries in complex, nested systems; and (e) deciding what criteria to use in answering questions about a particular boundary type. To facilitate the above I provide general criteria one may use for identifying ecological entities. Such criteria should assist in focusing on boundaries appropriate for a given research question. Finally, where advancing the theoretical framework for ecological boundaries is concerned, the diversity of boundary types will be better served when reorganized in relation to the concept of entity as discussed below.
\end{abstract}

1

Ecologists assume the existence of boundaries (Taylor, 2005), believe that they are important and ubiquitous in nature (Laurance et al., 2001) and contend that they should be studied. Typically ecologists ask how boundaries isolate habitats or organisms; how they regulate the transfer materials, organisms, or information; how selectively they perform these roles (filter); and ultimately how the presence and characteristics of boundaries affect neighboring areas or systems. Presumably, these questions only gain value and clarity when the ecological objects that are bounded are adequately specified. However, clear articulations of what objects and their boundaries are, whether in general terms or in specific cases, are infrequent and, when provided, may remain unsuitable for broader use across a diversity of ecological situations (see Strayer et al., 2003, for constructive observations). A partial fault may lie with the missing component - insufficient specification of objects separated by a boundary. This is because choosing boundaries without specifying objects can lead to choosing the wrong boundaries for the research question. This occurs for example when an observed boundary (e.g., physical barrier) is assumed to be important to an ecological process or variable, but examination of entities would show that it is unimportant at the scale studied.

To address this deficiency, the paper argues for a view of ecological boundary as an attribute of ecological system (or entity from now on). The preference for entity over other terms is that the meaning of entity entails a degree of autonomy and self-maintenance (cf. Kolasa and Pickett, 1989), regardless of other applicable connotations. The paper proposes that, being attributes of entities, boundaries are most effectively investigated (i.e., with maximum accuracy and minimum subjective guesswork) in relation to the entity they belong to. This proposition requires an explanation of what ecological entities are, the circumstances that lead to their formation, the universal properties they possess, and how this knowledge may broaden our understanding of boundaries. 
Following this core exposition, I then review how this perspective helps to identify and study boundaries, how it accommodates differences in scale, and what limitations and promises it has. In a nutshell - (1) the concept of boundary is inseparable from the concept of ecological system; (2) the ability to define the system determines the ability to define the boundary; (3) boundaries appear at different scales on account of the hierarchical structure of ecological systems; and (4) boundaries have different forms and diverse consequences (Strayer et al., 2003) as a direct result of the diversity of subsystems constituting a hierarchy (e.g., Jax et al., 1998).

Although many authors make general references to boundaries, only few explicit definitions are available. In a series of papers, Cadenasso et al. (2003a, b) and Strayer et al. (2003) contributed to the understanding of boundaries by both reviewing their kinds and attributes and offering a definition. Specifically, Cadenasso et al. (2003a) defined boundary as the zone of contact that arises whenever an area is partitioned into contrasting patches. The authors also recognize that entities play a role in defining boundaries when they refer to the contrasting patches as bounded systems. However, they do not elaborate on this aspect in depth.

The most recent definition of ecological boundary has been offered by Yarrow and Salthe (2008). The authors appear to pick up the concept where Cadenasso et al. (2003b) left it out and become more explicit about the necessity to recognize the requisite role of a system: they view boundary as a surface associated with an ecological system. Further, they also recognize two aspects: (a) that systems are hierarchical, that is, composed of and included in other systems, each with its own boundary, and (b) that a boundary must enclose "internal discernible" processes. They believe that without meeting this second condition, the boundary would not have functional meaning. The first aspect is shared with this paper, and the second aspect shows affinity to the concept of integration used here.

The idea that ecological entities are pivotal to various research pursuits is not new. Ecologists have long recognized that many questions can or must be handled within or related to specific ecological units bounded in space or time (e.g., Cousins, 1990; Taylor, 2005; Pfeifer et al., 2007). Being identifiable and discrete, these bounded units may be termed entities. Several examples of direct and indirect commitment to entities are useful. Lindeman (1942) stated this explicitly: "An ecosystem composes of physical-chemicalbiological processes active within a space-time unit" even though he gave no hint what that unit might be and how it could be identified. In contrast, Post et al. (2007) propose that the key to identification of communities and ecosystems lies in defining their boundaries. These differences in perspectives and emphases underscore the lack of clarity as to the identification of units that show boundedness, and hence underpin the idea of boundary, which continues to be a problem. For example, a general view is that ecologists define communities as groups of organisms living in the same place at the same time (Fauth et al., 1996). But by leaving criteria of where the (same) place begins and ends and how large it is out from this definition, community and ecosystem ecologists have little choice but to make arbitrary and possibly erroneous decisions about community boundary. In fact, any number of community boundaries might be chosen based on different criteria. One of the most discussed theories of species diversity, the neutral theory of biodiversity by Hubbell (2001), has as its central notion the zero-sum assumption. This assumption posits that an individual of any species can be added to the community if another individual is removed through death or emigration. The zero-sum assumption is an implicitly entity-oriented concept because it relies on counting individuals contained in a specified area.

One reason for why ecologists stop short of elaborating further on the concept of boundary is that they seem to assume that the boundaries are fairly obvious. But the practices of choosing boundaries are inconsistent and, at times, arbitrary. An example of such practices comes from informal and practical approaches to ecosystems that advocate drawing research-centered boundaries (e.g., Hau and Bakshi, 2004), with focus on the specific ecological problem but without an interest in the properties of the boundary itself. Others focus on natural patterns but do so in an ad hoc manner. For example, Krause et al. (2003) interpret the separation of compartments in food webs as reflecting "sutures" (boundaries) between different habitats. In this case, understanding compartmentalization in a food web will largely depend on the existence and understanding of gradients among habitats and differences among constituents of the food web. This seems to leave the rigor of food web analysis at the mercy of subjective decisions about sutures. Some ecologists have been troubled by the desire for good criteria for delineating communities (e.g., Wiens and Milne, 1989; Cousins, 1990) and ecosystems (Levin, 1992; Post et al., 2007).

The sample of literature above suggests that the existence of ecological entities plays a role in pursuing research questions, even when the entities are not clearly articulated and defined. When ecologists do specify entities, they often do so arbitrarily by adopting convenient and specialized criteria. For example, the watershed ecosystem approach, metapopulation and metacommunity, or population genetics studies all rely on spatially delineated aggregates of organisms and assume that these aggregates possess some distinguishing structural and functional attributes that apply to all variables of interest. Arbitrary does not mean erroneous but provides little assurance of correctness. More is needed.

In some areas of ecology, particularly ecosystems science, advances were made often without concern for spatial boundaries. Yet, even concepts such as ecosystem functions that are formulated without references to boundaries would face fewer limitations if boundaries or the corresponding entity were more explicitly specified. Ecosystem "functions" are quantified, modeled, and often presented as "services". 
However, an ecosystem function, here meant as a process (see Jax, 2005, for review of different meanings of function), would be difficult to examine without relating it to an entity that carries or performs that function. Detached from an entity, the notion of "function" as well as its evaluation would otherwise keep changing in response to spatial and temporal scale of data collection and would remain, both logically and practically, undeterminable. A sounder, less shifting notion of function arises from another use - as a contribution of one part (entity) to the rest of the system (cf. Jax, 2005). An analogy reveals the following distinction: a unicycle wheel (a component entity) is to move the device (the higher level entity) along the road (a contribution, i.e., function), but one half of the wheel has no such function, although it might have another function such as stopping the bike from rolling downhill. Irrespective of the particular use of "function", it is uncontroversial that change of spatial, temporal, or organizational scale matters to its interpretation, and it will thus matter to the function of a boundary.

An obvious but not trivial challenge for effective tackling of boundary-related questions is that boundaries appear to occur at any scale one looks at and that boundaries may contain, fully or partially, other boundaries. Considering just the zonal structure of a lake ecosystem, one sees a boundary between a lake and surrounding land habitats, between aquatic vegetation (which on one side shares the boundary with the lake) and bare lake bottom area, emergent vegetation (which on one side shares the boundary with the lake and with submergent vegetation on the other), and submergent vegetation (which on one side shares the boundary with emergent vegetation and the vegetation in general on the side of bare bottom area). Such nested overlaps and sharing of boundaries seem universal in nature.

Although discussion of entities and their relationship to boundaries is limited in the literature, systematic attempts to address the question of boundaries do exist. Cadenasso et al. (2003a) define boundaries via a pattern only as "areas of transition, contact, or separation between the contrasting elements of a mosaic". While this formulation appears to stress the pattern, in the next paragraph they go on to say "that boundaries are zones of transition between contrasting systems" - an acknowledgement of the importance of systems (= entities) in the process of defining a boundary. I expand on this perspective below and use some examples in support of the idea that the absence of clarity as to what those "systems" are may lead to ineffective or even misdirected research effort. Also, the lack of sound framework for defining boundaries carries risks of accumulating observations that are difficult to organize, generalize, and used in building a theoretical framework from. Relevant questions thus arise. How do we know which processes warrant investigation with respect to a specific boundary, particularly if the boundary is shared by large patches and their constituent smaller patches? Can we conceptualize and investigate boundaries when they leave no imprint on the environment, such as boundaries between "cultural" clans of song birds (see later on for more detail)? How do we navigate and appropriately change our specific research questions as we change the scale of observation at which we perceive patches and contrasts between them?

To deal with the questions and associated ambiguities mentioned above, we may need a second look at the notion of boundary itself. The goal of this paper is to aid in tightening foundations for a theoretical framework needed for studying boundaries. I believe that such a framework will be sound if the link between the boundary and its entity is made explicit.

In summary, literature perusal indicates that boundaries are deemed important in analysis of ecological patterns and processes and that they are, albeit only vaguely, associated with the notion of some kind of entity or system. Entities received less systematic attention, even though they play a more fundamental role in ecological research. In the next step, I outline a more restricted view of boundary and of the revealing relationship between it and the entity it belongs to.

\section{What are entities and how do they form?}

Because I contend that boundaries are derivatives of entities, I start from a premise that the ecological universe we examine is made up of things or entities (Ulanowicz, 1997; p. 148) that are somewhat connected and somewhat disconnected but never fully disconnected or fully connected. This circumscribes the issue: identification of ecological entities is a matter of degree related to the degree of their discreteness. Some entities, depending on strength of internal connection as opposed to the connections they have with their putative counterparts (see Post et al., 2007), vary in the degree to which they appear as separate objects. But what are entities?

Entities are natural objects (cf. Kolasa and Pickett, 1989, for theoretical foundations) that stand out from the environmental matrix or are delineated from other objects through several traits. These include autonomy, persistence, internal coordination, and interdependence of parts that add up to a general characteristic: integration and wholeness. The traits vary from entity to entity both in their combination and magnitude, which produces different levels of integration and different capacity to appear as separate objects. Highly integrated entities are discrete and stand out very clearly from the matrix while poorly integrated entities intergrade with one another and are more difficult to discern (see also Sect. 2.1).

In addition to accepting commonality of entities, I assume the following here: (a) boundaries are where an entity ends (Yarrow and Salthe, 2008), (b) the entities and hence their boundaries vary in discreteness, and (c) evaluation of the degree of discreteness may require a range of methodologies. 


\subsection{A case for entities as the fundamental ecological unit in plain language}

If there were no entities, then for a given scale of resolution, the universe would look homogeneous and flat, although perhaps inclined on occasion (gradient of conditions). Entities imply heterogeneity within the universe of interest (e.g., landscape, metacommunity, population, or colony), but not just any kind of heterogeneity. They imply a particular kind of heterogeneity: one which can include a range of entities differing in integration. Because integration determines whether an observer perceives a portion of the universe as having a separate, autonomous existence (even if only slightly autonomous), heterogeneity is seen as a state of having areas of different levels of discreteness. When such existence is commonly recognized, ecologists attach terms such as subpopulation, local community (in a metacommunity), caste in a colony, or successional stage in a patchwork of vegetation types.

Does understanding how entities form assist in articulating questions about boundaries? Is it by knowing what class of boundary one is dealing with? And does knowing what class it belongs to say something about the mechanism? I suggest that the entity formation involves three types of cases: (a) biological interactions alone; (b) biological interactions modulated by environmental differences (which could be gradual or abrupt); and (c) physical environmental constraints acting directly.

\subsubsection{Biological interactions alone - examples}

Two situations below represent a broad range of conditions and ecological levels of organization where biological interactions lead to emergence of entities or, in other words, a localized degree biological self-organization. Ant colonies (or termites, wolf packs, lion prides, shoals of fish, mixed herds of grazers) form entirely through interactions among participating individuals. The underlying mechanisms are genetic and behavioral. Many of such entities create a distinct spatial signature on the landscape, but not all of them do. Nor do all mechanisms underlying emergence of biologically determined entities involve genetic and behavioral controls: at the other end of the spectrum, a chance and uneven distribution of biological resources may produce partly autonomous entities. If two or more species (often plants and their consumers) interact in ways that lead to local persistence of the actors, pockets of increased integration will emerge such as close links between plants and soil organisms (Bertness and Callaway, 1994; Wardle et al., 2004). Coral reefs, vegetation near prairie dog towns or termite nests (Brody et al., 2010; Davidson et al., 2012; Fox-Dobbs et al., 2010), or selfmaintaining alternative states (e.g., Beisner et al., 2003) illustrate how species interactions can form local entities. In general, positive interactions appear to play a decisive role in the formation of community-level entities (cf. Bruno et al., 2003).

\subsubsection{Environmentally modulated entities}

This category includes cases where physical environment does not impose limits on the extent of an entity but where a shift in the physical regime mediates the decisive role of biotic interactions. Here an entity forms through an interaction between an environmental gradient and a differential use of it by individuals, species, or communities. Zonation of barnacles on a rocky coast (e.g., Okuda et al., 2010) exemplifies this common mechanism, which might be expected whenever a change in physical conditions leads to a shift in competitive abilities, vulnerability to predators and pathogens, or in reproductive output. A general theoretical exploration of how identifiable communities form through such a process in a metacommunity is available in Filotas et al. (2010). While swapping dominance between species on an environmental gradient is not synonymous with emergence of a new entity, an emergence of a new dominant species (e.g., a different barnacle species) is likely to induce a shift in composition of associated species and a new set of interactions among them - all likely ingredients for a separate entity.

\subsubsection{Physical environmental constraints}

The third type of situation allowing a set of organisms to live and interact together as a discrete entity can emerge when physical conditions in space exceed physiological tolerance of species, their ability to reproduce successfully, or to migrate somewhere else. Ecological entities defined through such constraints map well onto the texture of the physical landscape. Examples span a broad range of ecological circumstances, from pitcher plant communities, small fish populations restricted to a desert spring (see Kodric-Brown and Brown, 1993), wetlands, riparian systems in arid areas to insular co-evolving florae and faunae (e.g., New Zealand or Lake Baikal). Food web compartmentalization is another area of ecology where strong habitat differences are seen to lead to spatially discrete networks of interactions (Power, 2006).

Prior to studying boundaries, ecological entities may be identified and characterized using a comprehensive set of methodological tools at ecology's disposal. A few general steps might help too, though. These steps are rooted in the idea that components of an entity are more integrated with each other than with similar components of another entity. Integration means that these components interact more often with each other, that the majority of their interactions are directly or indirectly positive (i.e., contributing to the persistence of the components), and that they involve a measurable degree of reciprocity.

When viewed as spatial, temporal or otherwise ordered series, differences in integration form a landscape of peaks and 
valleys, with peaks being diagnostic of separate entities (Kolasa, 2005). The following gives suggestions for how to find or map areas of that landscape where the relatively elevated integration occurs:

a. Choose your focal entity, e.g., a colony of ants, a local population of voles, a metacommunity of pitcher plant invertebrates, or a lake ecosystem, to name a few.

b. Choose/decide on criteria you wish to apply to determine the extent of the selected entity. These criteria must be appropriate (based on the best current information and be suitable for the entity of interest), and their quantitative expression should closely match that implied by the research question. For example, in the case of the ants it may be relatedness of ants within the colony or area the colony controls. Other entities will call for other criteria. For example, a metacommunity might be defined by higher rates of dispersal among a cluster of patches as compared to other clusters in the general neighborhood. See additional examples in Table 1 .

c. Obtain and analyze data to determine the spatial (or organizational) extent over which elevated integration occurs and where it drops. The extent of the elevated integration defines the bounds of the entity of interest.

Once the criteria listed in Table 1 are evaluated, boundaries emerge as unambiguous limits of the focal entity. These criteria will require development and vetting for each hypothetical entity and may be used individually or in combinations that are most likely to discriminate among candidate entities or an entity and the background template. While operational issues will never go away, the conceptual issues of study design (including boundary questions) become much more manageable. An example follows.

Consider an ant colony and two different ways (criteria) of describing it. A genetic criterion of relatedness delineates the colony from all other colonies by being $75 \%$ related among all its female members (in haplodiploid species, females develop from fertilized eggs and males develop from unfertilized eggs. Thus, daughters will share $100 \%$ of fathers' genes and $50 \%$ of their mothers' and therefore $75 \%$ of their genes with each other). The relatedness between the two most related colonies cannot be higher than $62.5 \%$ on average if they have two sisters as mothers and the same father and just $12.5 \%$ for two unrelated fathers. Thus, the colony organizational extent is defined by inclusion of all highly related individuals. Spatial extent of ant activity matches completely the genetic relatedness of ant. However, when scent marking of foraging area is the proxy measure of activity, the colony extent becomes less accurately assessed because the scent markings change over time in response to changing activities and scent dissipation. A colony so described may in fact overlap partly with another colony whose members make forays into the focal colony territory, at least on some occasions. Consequently, both criteria eliminate ambiguities as to the identity of a colony, but an inadequate timescale at which data are collected may introduce a degree of vagueness as to its extent (because scent marking is only an approximate measure and a transient imprint of the entity on the landscape), the latter being an operational issue only. Similarly, the relatedness criterion does not account for related individuals that separated from the main colony, nor for the fact that the queens of adjacent colonies might be sisters (however, both problems are easy to correct by adjusting the timescale or range of expected relatedness values). In spite of these minor hurdles, both criteria are capable to identify and separate the colony from other colonies exactly, even if their territories overlap. The operational aspects may be not as easy as for the ant colony when communities are considered but the anchoring the investigation in the concept of entity should help nevertheless.

\section{Entity and its relation to boundary}

In line with the earlier statement, boundaries represent a phenomenon and they do not have independent existence but are products of a relationship between two adjacent entities or of an entity and its surroundings. A somewhat different view was taken by Yarrow and Salthe (2008), who allow for giving the boundary independent status (i.e., as a separate entity). Nevertheless, this paper's thesis implies that there is no boundary between a forest patch and a grassland if the forest is missing and no boundary between ant colonies without ants. This further means that identification of boundaries, from the logical standpoint, requires identification of entities. The hierarchical nature of entities is a major characteristic that affects understanding of boundaries: as entities are hierarchical (Kolasa and Pickett, 1989; Cadenasso et al., 2003a; Allen and Holling, 2008), so are boundaries. This further implies that

a. boundaries tend to become more diffuse and less defined as integration - and thus discreteness - decreases. And, because integration declines with increasing scale (organizational level of entity), so too does boundary definition in terms of organization or space;

b. identifying entities from boundaries alone is unreliable because more than one boundary of similar material expression but belonging to different entities may exist at several scales of resolution;

c. analysis of boundaries carries risks of mixing their different types in the analysis whenever entities are inadequately specified (i.e., boundaries produced by entities between which essential differences were not recognized); 
Table 1. Entities and possible criteria that could be used in defining them (examples). The criteria here are for the sake of argument rather than supplying a particular area of research with solutions. They aim to show that entities can be defined without employing the concept of boundary even if boundaries, once determined, may be useful in identifying entities.

\begin{tabular}{|c|c|c|}
\hline $\begin{array}{l}\text { Entity type } \\
\text { (examples) }\end{array}$ & Applicable criteria & $\begin{array}{l}\text { Indicators of elevated integration (to be } \\
\text { interpreted in contrast to other candidate entities) }\end{array}$ \\
\hline \multirow[t]{4}{*}{ Ant colony } & - relatedness & High relatedness among colony members \\
\hline & - spatial proximity of components (individuals) & High average proximity \\
\hline & - ability to survive independently & Low ability to survive independently of the rest of colony \\
\hline & - individual recognition & Colony members can identify each other as such \\
\hline \multirow[t]{4}{*}{ Local vole population } & - probability of encounters among individuals & High \\
\hline & - relatedness/or recognition & $\begin{array}{l}\text { High on average among population members relative } \\
\text { to non-members }\end{array}$ \\
\hline & - population size & Viable if isolated \\
\hline & - population density & Elevated \\
\hline \multirow[t]{2}{*}{$\begin{array}{l}\text { Metacommunity of pitcher } \\
\text { plant organisms }\end{array}$} & - dispersal & $\begin{array}{l}\text { High among patches belonging to a given } \\
\text { metacommunity }\end{array}$ \\
\hline & - participation in one food processing chain & Shared (locally)* \\
\hline \multirow[t]{3}{*}{ Lake ecosystem } & - spatial coherence of components and processes & High relative to that between lake ecosystems \\
\hline & - nutrient cycles & Fairly complete \\
\hline & - available energy pool & Shared among components \\
\hline
\end{tabular}

* Note that metacommunity may comprise nuclear communities or groups of such communities.

d. when different entities encounter environmentally modulated discontinuities (vegetation edges, geological and hydrological discontinuities), their boundaries may align together and thus pose a challenge to analysis of their individual properties and effects;

e. because entities behave so, boundaries can form at any point of a gradient from pure environmental drivers to biological interactions alone (see section on the formation of entities).

Below, I make additional comments on the items above. Entities differ in their integration or an overall degree of cohesion and interaction strength among entity components. Because of the defining role of entities, the strength of positive interactions (connections) among components of an entity decides how conspicuous and sharp its boundary appears. Within this framework, a zone of low connection strength around a core of higher connection strength emerges as the entity end and thus a boundary. This zone may have an obvious and tangible form or may be virtual in nature as in territorial borders between nesting bird pairs. As in the latter no physical boundary is present, the boundary is delineated by a drop in bird-to-bird cooperative interactions and, in some cases, by an increase in antagonistic interactions. The presence of physical discontinuities, or discontinuities due to other biological processes, may reduce interaction strength among ecological objects and thus aid in formation of separate entities and ensuing boundaries. In such cases environmental discontinuities create and coincide with at least some boundaries (as suggested by case "c" in the section on ways by which entities form).
Another implication of linking boundaries to entities relates to the former being seen as filters (Fagan et al., 2003) between an entity and its neighbors or broader environmental matrix. These filters affect the rate and quality of the exchange of materials and information between adjacent entities. The strength of boundaries and hence their role as filters can vary, and it generally declines with the increase in the size of the system and concomitant decline of its internal integration.

Discreteness of entities affects the nature and perception of boundaries. Once entities are identified at the scale of interest, the boundaries between them are exposed, but no more than discreteness of the respective entities permits. However, entities at a higher level of organization - those containing other component entities - are as a rule less integrated (Kolasa and Pickett 1989) and hence more difficult to conceptualize and quantify. Furthermore, entities at several organizational levels may possess boundaries of similar material nature. When entities are nested, these boundaries are superimposed. Here, the combined complexity of scaling and nestedness challenge boundary detection and the discovery of the ecological consequences of these boundaries. A graphical summary (Fig. 1) exposes some of the consequences.

One of these issues is methodological. While projecting entities onto a two-dimensional space leaves an imprint of boundaries (Fig. 1c), it would be difficult if not impossible to reconstruct the nature and arrangement of entities that formed it. This difficulty would become clear if panels $1 \mathrm{~A}$ and $1 \mathrm{~B}$ were removed. The reverse mapping, from pattern to entity, becomes less reliable than from entity to pattern. For example, at coarse analytic resolution, two ant colonies might overlap in the use of space, which would carry a risk 


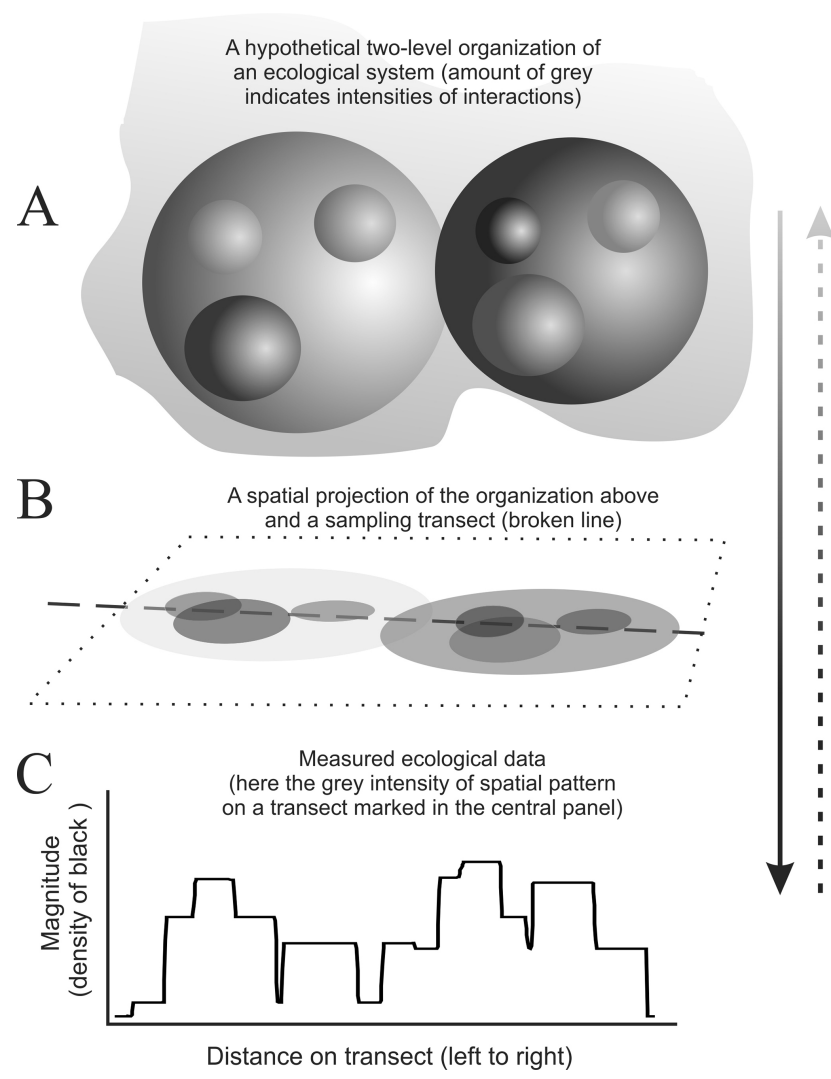

Figure 1. Relationship between entities, their boundaries, and a pattern (of black ink in this case). (A) a hypothetical two-level organization of an ecological system (amount of grey symbolizes internal strength of interactions; (B) spatial projection of the organization above and a sampling transect (broken line); (C) typical ecological data (here the data are density of black pigment on a transect marked in (B). Note that a progressive loss of boundary discreteness expected in higher level entities is not shown in (C). The continuous arrow on the right indicates direction of successful mapping. The broken line indicates direction of increasing difficulty (entities become more difficult to identify when one starts with the pattern only). Lighter shading of the line indicates increased ambiguity. Note that boundaries arise at different levels of organization, which allows for different processes, whether biological or geomorphologic, to be nested, to overlap, intersect, and to interact in complex and specific ways, depending on the system of interest. In this model, the tension between community based approach to boundaries and those associated with ecosystems (cf. Post et al., 2007) can in principle be reduced, if not entirely eliminated.

of assigning individuals of one colony into the other and so making reconstruction of an entity from an instantaneous record of spatial extent inaccurate. Indeed, a subsequent analysis of cooperation, food sharing, defense, and other biological processes would be seriously impaired if colony members were assigned to incorrect colonies. This illustrates asymmetry of the discovery process, which sets the limits on formulating questions about boundaries before reaching sufficient clarity as to which entities they pertain to. The task be- comes even more difficult when boundaries have more complex shapes such as disjunct, diffuse, or mosaic (cf. Schaefer et al., 2011; Kolasa and Zalewski, 1995). Thus, although discontinuities in the pattern can suggest existence of entities (cf. Bandelj et al., 2012, for recent efforts on detecting hierarchically arranged metacommunities and their boundaries), they do not define those entities consistently. Boundaries defined by entities do not suffer from this serious problem.

Admittedly, discontinuities in pattern may be easier to detect than the entities that produce them (see for the range of methods for the former in Fortin et al., 2000), and thus the detection of discontinuities can serve, when used with caution, as a tool to construct a tentative, possibly testable, view of entities that underpin them.

The general view proposed here - using entities to ground ecological investigation - offers another advantage. It allows boundaries to be seen even when contrasts between patches are absent (think of two adjacent ant colonies), when discontinuities in habitat attributes are absent, and when no sharp transitions take place. For example, sharp boundaries will not be found whenever a transition from one entity to another is gradual. Thus, logical clarity does not guarantee the ability to draw a well-defined boundary between two entities as may be required for sampling design and statistical analysis. Nevertheless, logical clarity provides a sound basis for empirical refinements, or arbitrary decisions if necessary: in colonial corals it is easy to identify two adjacent polyps - the two entities. An investigator will have no doubt that, for some questions, these two polyps warrant a separate treatment. No amount of empirical work, however, is likely to find where a boundary between them is because of its gradual or diffuse nature. To set this boundary one could choose an equal distance from mouths of the polyps, or the point where ectodermal cells receive equal amount of nutrients from each polyp in question. Despite the arbitrariness of these criteria, the decision can be seen as sound because its conceptual basis (an assumption of two entities) provided a good starting point. Thus, broad, fuzzy, and gradual transitions should not a problem. Assemblages of organisms on individual patches of coral reefs (Fig. 2) illustrate another issue that is resolved by using ecological entities as criterion for identifying boundaries. Here, an ecological entity is discrete and well defined by the lifestyle of the species living on a patch reef. Many of these species, if not a great majority, live, hide, feed, and reproduce on the patch reef or in its immediate proximity (e.g., Green et al., 2012). Organisms living on a single patch form a distinct local community clearly separate from communities on neighboring patches. When a patch is far enough from other patches, the community boundary is emphasized by the contrast between area under the grazing pressure and the ungrazed area farther away. However, when the patches are closer and the grazing halos merge, the contrast disappears even as the assemblages remain distinct and their boundaries remain unchanged. To determine the boundary of assemblages enclosed within the merged halo areas, it 


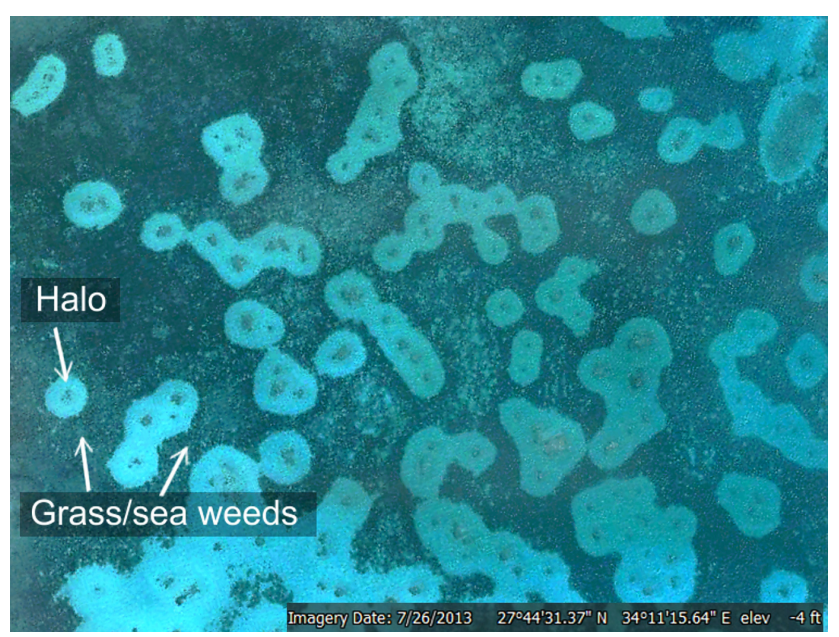

Figure 2. Coral reef patches surrounded by halos of sand produced by herbivore members of a patch assemblage (fish, mollusks, and sea urchins) who remove sea grass and algae. Boundary of the patch assemblage is visible where halo contrasts with the grass and algae area but requires different identification criteria when two or more halos meet. A typical single patch halo is about $25-45 \mathrm{~m}$ across, with a darker coral patch at the center ranging from $5-15 \mathrm{~m}$ in diameter. Image copyright $2014 \mathrm{CNES} /$ Astrium.

will thus be necessary to use an entity-oriented perspective and, for example, map activities of organisms associated with the local reef patch.

Because boundaries emerge between ecological entities that operate at a range of scales and because a range of different processes, mechanisms, and physical expressions can create boundaries, it is impractical to concentrate on specific methods for detecting boundaries. Further, because components at different levels of ecological organization differ in properties, their boundaries will have different material expressions. It is quite clear that different criteria would be used to find boundaries among clans of song sparrows, members of which sing differently and songs that reproductively isolate clans than among allopatric populations, e.g., wolf packs (Fig. 3) or among different watersheds. Similarly, different measurements at different levels of resolution may be needed to detect boundaries at different scales. Boundaries of communities in pitcher plants are discrete and easier to define than boundaries between pitcher plants (cf. Miller and Kneitel, 2005); a lion pride boundary is easier to define (in terms of membership or territory, whatever the focus) than that of a lion population. Similarly, wolves on Isle Royale in Lake Superior form one genetic unit (Adams et al., 2011), but this population of genetically related individuals split into two antagonistic packs. Thus, genetic boundaries may not coincide with ecological (or social, in the case of these wolves) ones even in a case that one would suspect such an overlap - a fact that is only revealed when employing entity-specific criteria and reviewing boundaries at several scales.

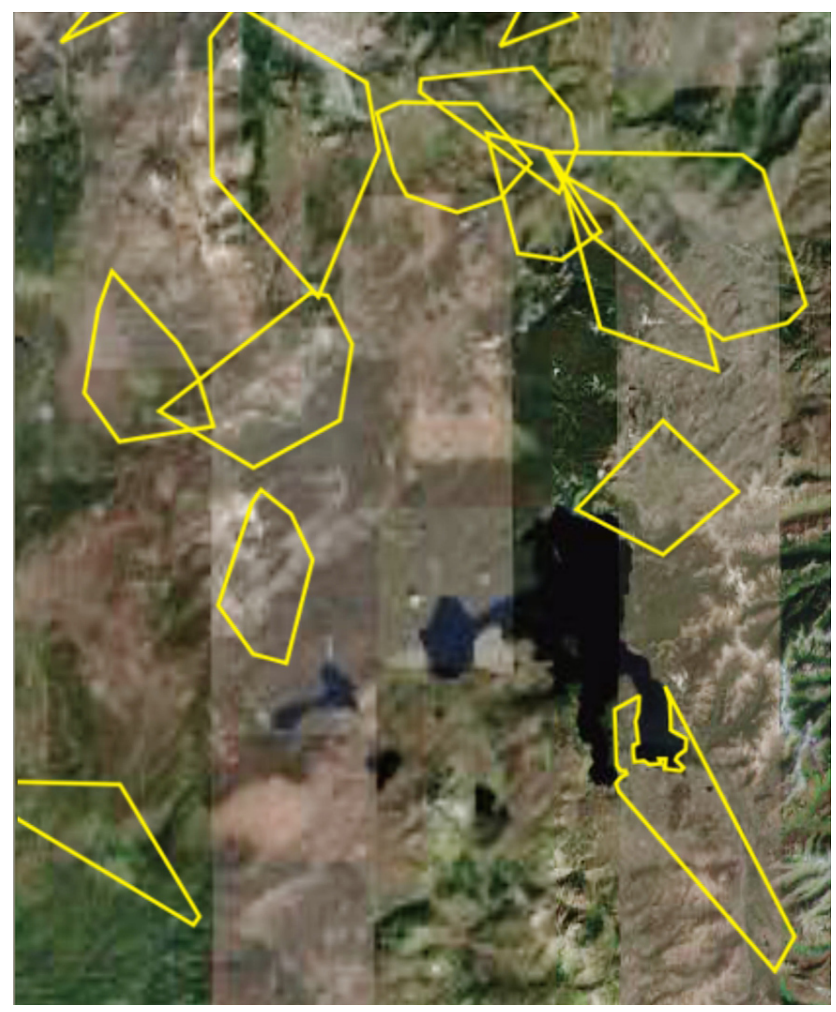

Figure 3. Wolf pack territories have boundaries (year 2004) that extend over different habitats and thus ignore the majority of habitat discontinuities. Territories may also overlap and boundaries intersect. This case illustrates that a search for "visible" or pattern-based boundaries has conceptual weaknesses and practicalities. Map of territories adapted from http://www.nps.gov/features/yell/slidefile/ mammals/wolf/Images/18175.gif

\section{Kinds of boundaries}

In line with the argument that boundaries are where ecological entities end or meet, I identify various kinds of boundaries as defined by the mechanisms that separate entities as well as by entities to which they refer. An excellent classification of boundaries (Strayer et al., 2003) often applies to and expands on the list below. Boundaries can take a great range of material forms and be created by a vast number of processes. Literature usage of the term "boundary" is quite diverse, too. Boundaries have been referred to as edges, ecotones, boundary layers, gradients, clines, transition zones, interfaces $(\mathrm{Ca}-$ denasso et al., 2003b), sutures, as well as species range limits (Maurer and Taper, 2002). Such a diversity may be seen as a testament to the absence of established theoretical framework with which to approach boundaries, although more recently Post et al. (2007) reviewed the relationship between the identification of ecosystems and the role of boundary in this effort. Post et al. (2007) also anchor their search for boundaries in the idea of entities (ecosystem in their case) that are defined by strong internal interactions. In this paper I generalize 
this to include all ecological entities. Using ecosystems, they focused on two general kinds of boundaries: structural and functional. Generalizing on their review, I suggest further examples from various areas of ecology, each with many permutations, and mixes of boundary-creating forces include the following:

a. "Cultural" boundaries similar to those that occur between subpopulations of song sparrows that produce songs in different dialects. Individuals select breeding partners on the basis of their song similarity and inherit the song dialect from their parents. The combination of the preference and inheritance forms an effective barrier among groups of individuals that otherwise face no other obstacles to interbreeding. These kinds of boundaries are likely to occur within communities and ecosystems.

b. Territorial boundaries between territorial individuals or groups (prides of lions, wolf packs, individual cats, families of bee-eaters) remain enforced by antagonistic behaviors and often involve ritualized threats and physical markings such as scent or scratches. As cultural boundaries above, they do not appear to separate communities or ecosystems but form within those entities. The above applies only to the most popular uses of the term community and ecosystem. Communities may indeed form within territories: invertebrate inhabitants of a bird nest can be treated as a community even though they form it within a bird territory, but such a view does not undermine the general concept of territorial boundary here.

c. Range boundaries containing migratory populations and maintained by a combination of bounding factors some of which may include geographical obstacles, seasonality, distribution of resources, or historical patterns remembered by individuals with migratory experience or encoded genetically as instincts. Migration ranges are likely to include more than one community and/or ecosystem. It is possible to consider the ecological entities spanned by a migration range of some species as higher level systems. For example, the Serengeti can be seen as a large ecosystem integrated by moving populations of large grazers. At the same time, the Serengeti includes many smaller and better defined ecosystems (Gillson, 2004). The relationship between the community/ecosystem and the organisms operating at broader spatial or temporal scales has been recognized as difficult one, and not yet resolved (O'Neill, 2001). Nonetheless, the hierarchical view that allows smaller ecosystems be part of a larger one might offer a solution (see further below).

d. Boundaries among associations of individuals caused by biotic interactions such as predation, mutualism, or competition. Examples include empty sand halos around patch coral reefs caused by predation on algae by reef residents, or zones of organisms formed along physical gradients such as those present on mountain slopes or rocky shores. Similar boundaries may also form through indirect effects of interactions between physical and biotic processes. For example, stream damming by beavers creates ponds and wetlands clearly delineated from the surrounding forest largely because of changes in hydrology. However, such boundaries are not conceptually different from those listed in the next item. They deserve a distinction because of the tradition of ecological research more than because of being a separate category.

e. Boundaries among communities and ecosystems. These boundaries often form in response to physical landscape features that disrupt continuity of constituent populations and lower the intensity of interactions among them. In some cases such as coral reefs, or habitats with important ecosystem engineers (e.g., beavers, prairie dogs), biological activities make mechanism attribution unclear. Nevertheless, topographical, hydrological, geological, or climatic variation in a landscape separates groups of species and thus contributes to partial differentiation of composition, interactions, and dynamics within such groups. The abrupt nature of change in physical features inevitably coincides with change in the flux rates at which matter, materials, and individuals exchange across gradients that form along physical transitions. Once separate ecosystems are established, internal processes may contribute to, enhance, and perpetuate the original discontinuities that initiated the emergence of separate ecosystems to begin with.

f. Boundaries between ecosystem complexes such as biomes. Such boundaries represent, consistent with the concept of biome, responses to edaphic and climatic conditions. Usually, they involve broad transition zones which pose the same classification difficulties as the separation among polyps of a coral colony. Categories appearing under heading "e" and " $\mathrm{f}$ " are mentioned here to conform to the prevailing uses in ecology rather than to claim well-defined differences. Distinctions about communities, ecosystems, and biomes are contingent on scale, research conventions, and methodological choices. Abandoning this terminology and replacing it with the concept of entity defined by attributes mentioned earlier (Table 1) may be ultimately more useful in study of boundaries. For the moment, I keep the conventional distinctions for the sake of familiarity.

One might be tempted to identify boundaries whenever contrasts exist between adjacent patches (assumed to be different entities). For example, Chust et al. (2003) developed a multilevel approach to quantifying the landscape grain based 
on the concept of contrast, that is, the magnitude of difference in measures across a boundary between adjacent patch types. This approach may be effective under some circumstances but is unlikely to work for situations where entities are similar yet distinct (i.e., separated by decrease in interaction strength or by antagonisms), such as between two adjacent plants of the same species or two prides of lions.

Based on this brief overview, it is possible to identify major ways in which ecologists have attempted to approach boundaries. So far, I identified at least three themes or approaches to boundary recognition: (i) steepness of a gradient in a variable, (ii) the amount of contrast between adjacent patterns, and (iii) entities as "owners" of boundaries. Post et al. (2007) provide a more concrete listing of specific physical and biological processes typically associated with either structural or functional boundaries, although their immediate focus is limited to ecosystems.

\section{Discussion and summary}

The concept of boundary becomes clear in conjunction with the concept of entity (system). A general unification offered by this view may be particularly useful given the diversity of material differences among boundaries. The ability to define the entity determines the ability to define the existence and properties of the boundary (and vice versa) within a reference framework. Because ecological systems have hierarchical structures, boundaries among components will appear at different observer scales (see Strayer et al., 2003, for considerations of grain and extent; and Yarrow and Salthe, 2008, for an excellent account of the hierarchical nature of boundaries).

Because entities at different levels of ecological organization differ in properties, their boundaries will have different material expressions and so origin, properties, and associated mechanisms. Nevertheless, keeping primary focus on entities should improve tracking and comparing those differences in unambiguous ways, and thus offer an advantage over the current practice. Consequently, a productive research program outlined by Cadenasso et al. (2003a), with focus on causes and forms of ecological boundaries, should benefit from recognition that this material diversity of boundaries can be seen through a lens of conceptual unity. Only then, after the boundary between entities has been specified, the variables and the observations scales most appropriate for the research question can adequately quantify, fully and effectively explore, and help interpret the role of boundaries (see Laurance et al., 2001).

Depending on the nature of the system, boundaries can be discrete or diffuse (recall the difficulty of determining where one coral polyp ends and another begins). When boundaries form on continuous environmental gradients, this particular indeterminacy may take the form of intergrading, or gradual change from one entity to another, especially at larger spatial scales where entity integration is weak. Furthermore, because external conditions and state of entities change over time, so do the boundaries: they are dynamic - their location, discreteness, and strength will vary. Finally, because the conceptualization and detection of entities depends on the scale of observation and processes under consideration, boundaries are also scale-dependent. This may imply that a search for boundaries may require different types of data depending on whether one looks, for example, at a local population or at its subunits (such as it social groups). Keeping an eye on the entity as a prerequisite for deciding on the relevant questions about its boundary should help in navigating through the multitude of boundary types and aspects such as origin, structure, shape, functions, and dynamics.

Ecologists, whether they recognize it or not, loosely think in terms of entities. So it is logical to define research approaches in those terms, and follow the same general criteria and rules for entity detection whenever boundaries become the focus of attention. In closing, I reiterate that advancing theoretical approach to boundaries requires refocusing on ecological entities. Entities define the appropriate scale for studying boundaries, entities are recipients of transfers, filtering, and separation that boundaries engender, and entities provide guidance as to which kind of boundary is relevant, irrespective of whether it is conspicuous or not.

Acknowledgements. I wish to thank A. Hayward and Matthew Hammond for numerous constructive suggestions on a draft of this text as well as Dr. V. Grimm who was one of the reviewers. Natural Research and Engineering Council of Canada provided funds.

Edited by: K. Jax

Reviewed by: two anonymous referees

\section{References}

Adams, J. R., Vucetich, L. M., Hedrick, P. W., Peterson, R. O., and Vucetich, J. A.: Genomic sweep and potential genetic rescue during limiting environmental conditions in an isolated wolf population, P. Roy. Soc. B-Biol. Sci., 278, 3336-3344, 2011.

Allen, C. R. and Holling C. S.: Cross-scale structure and the generation of innovation and novelty in discontinuous complex systems, in: Discontinuities in ecosystems and other complex systems, edited by: Allen, C. R. and Holling, C. S., Columbia University Press, New York, 2008.

Bandelj, V., Solidoro, C., Curiel, D., Cossarini, G., Canu, D. M., and Rismondo, A.: Fuzziness and heterogeneity of benthic metacommunities in a complex transitional system, Plos One, 1-15, e52395, doi:10.1371/journal.pone.0052395, 2012.

Beisner, B. E., Haydon, D. T., and Cuddington, K.: Alternative stable states in ecology, Front Ecol. Environ., 1, 376-382, 2003.

Bertness, M. D. and Callaway, R.: Positive interactions in communities, Trends Ecol. Evol., 9, 191-193, 1994.

Brody, A. K., Palmer, T. M., Fox-Dobbs, K., and Doak, D. F.: Termites, vertebrate herbivores, and the fruiting success of Acacia drepanolobium, Ecology, 91, 399-407, 2010. 
Bruno, J. F., Stachowicz, J. J., and Bertness, M. D.: Inclusion of facilitation into ecological theory, Trends Ecol. Evol., 18, 119125, 2003.

Cadenasso, M. L., Pickett, S. T. A., Weathers, K. C., Bell, S., Benning, T. L., Carreiro, M. M., and Dawson, T. E.: An interdisciplinary and synthetic approach to ecological boundaries, Bioscience, 53, 717-722, 2003a.

Cadenasso, M. L., Pickett, S. T. A., Weathers, K. C., and Jones, C. G.: A framework for a theory of ecological boundaries, Bioscience, 53, 750-758, 2003b.

Chust, G., Pretus, J. L., Ducrot, D., Bedos, A., and Deharveng, L.: Identification of landscape units from an insect perspective, Ecography, 26, 257-268, 2003.

Cousins, S. H.: Countable ecosystems deriving from a new food web entity, Oikos, 57, 270-275, 1990.

Davidson, A. D., Detling, J. K., and Brown, J. H.: Ecological roles and conservation challenges of social, burrowing, herbivorous mammals in the world's grasslands, Front. Ecol. Environ., 10, 477-486, 2012.

Fagan, W. F., Fortin, M. J., and Soykan, C.: Integrating edge detection and dynamic modeling in quantitative analyses of ecological boundaries, Bioscience, 53, 730-738, 2003.

Fauth, J. E., Bernardo, J., Camara, M., Resetarits, W. J., VanBuskirk, J., and McCollum, S. A.: Simplifying the jargon of community ecology: A conceptual approach, Am. Nat., 147, 282286, 1996.

Filotas, E., Grant, M., Parrott, L., and Rikvold, P. A.: Positive interactions and the emergence of community structure in metacommunities, J. Theor. Biol., 266, 419-429, 2010.

Fortin, M. J., Olson, R. J., Ferson, S., Iverson, L., Hunsaker, C., Edwards, G., Levine, D., Butera, K., and Klemas, V.: Issues related to the detection of boundaries, Landscape Ecol., 15, 453-466, 2000.

Fox-Dobbs, K., Doak, D. F., Brody, A. K., and Palmer, T. M.: Termites create spatial structure and govern ecosystem function by affecting N-2 fixation in an East African savanna, Ecology, 91, 1296-1307, 2010.

Gillson, L.: Evidence of hierarchical patch dynamics in an east African savanna?, Landscape Ecol., 19, 883-894, 2004.

Green, B. C., Smith, D. J., and Underwood, G. J. C.: Habitat connectivity and spatial complexity differentially affect mangrove and salt marsh fish assemblages, Mar. Ecol. Prog.-Ser., 466, 177192, doi:10.3354/meps09791, 2012.

Hau, J. L. and Bakshi, B. R.: Promise and problems of emergy analysis, Ecol. Model., 178, 215-225, 2004.

Hubbell, S. P.: The Unified Neutral Theory of Biodiversity and Biogeography, Princeton University Press, Princeton, 2001.

Jax, K.: Function and "functioning" in ecology: what does it mean?, Oikos, 111, 641-648, 2005.

Jax, K., Jones, C. G., and Pickett, S. T. A.: The self-identity of ecological units, Oikos, 82, 253-264, 1998.

Kodric-Brown, A. and Brown, J. H.: Highly structured fish communities in Australian desert springs, Ecology, 74, 1847-1855, 1993.

Kolasa, J.: Complexity, system integration, and susceptibility to change: Biodiversity connection, Ecol. Compl., 2, 431-442, 2005.
Kolasa, J. and Pickett, S. T. A.: Ecological systems and the concept of biological organization, P. Natl. Acad. Sci. USA, 86, 88378841, 1989.

Kolasa, J. and Zalewski, M.: Notes on ecotone attributes and functions, Hydrobiologia, 303, 1-7, 1995.

Krause, A. E., Frank, K. A., Mason, D. M., Ulanowicz, R. E., and Taylor, W. W.: Compartments revealed in food-web structure, Nature, 426, 282-285, 2003.

Laurance, W. F., Pérez-Salicrup, D., Delamônica, P., Fearnside, P. M., D'Angelo, S., Jerozolinski, A., Pohl, L., and Lovejoy, T. E.: Rain forest fragmentation and the structure of Amazonian liana communities, Ecology, 82, 105-116, 2001.

Levin, S. A.: The problem of pattern and scale in ecology, Ecology, 73, 1943-1967, 1992.

Lindeman, R. L.: The trophic-dynamic aspect of ecology, Ecology, 23, 399-417, 1942.

Maurer, B. A. and Taper, M. L.: Connecting geographical distributions with population processes, Ecol. Lett., 5, 223-231, 2002.

Miller, T. E. and Kneitel, J. M.: Inquiline communities in pitcher plants as a prototypical metacommunity. In: Metacommunities: spatial dynamics and ecological communities, edited by: Holyoak, M., Leibold, M. A., and Holt, R. D., University of Chicago Press, Chicago, 2005.

O'Neill, R. V.: Is it time to bury the ecosystem concept? (With full military honors, of course), Ecology, 82, 3275-3284, 2001.

Okuda, T., Noda, T., Yamamoto, T., Hori, M., and Nakaoka, M.: Contribution of environmental and spatial processes to rocky intertidal metacommunity structure, Acta Oecol., 36, 413-422, 2010.

Pfeifer, M. A., Henle, K., and Settele, J.: Populations with explicit borders in space and time: Concept, terminology, and estimation of characteristic parameters, Acta Biotheor., 55, 305-316, 2007.

Post, D. M., Doyle, M. W., Sabo, J. L., and Finlay, J. C.: The problem of boundaries in defining ecosystems: A potential landmine for uniting geomorphology and ecology, Geomorphology, 89, 111-126, 2007.

Power, M. E.: Environmental controls on food web regimes: A fluvial perspective, Prog. Oceanogr., 68, 125-133, 2006.

Schaefer, J. F., Duvernell, D. D., and Kreiser, B. R.: Ecological and genetic assessment of spatial structure among replicate contact zones between two topminnow species, Evol. Ecol., 25, 11451161, 2011.

Strayer, D. L., Power, M. E., Fagan, W. F., Pickett, S. T. A., and Belnap, J.: A classification of ecological boundaries, Bioscience, 53, 723-729, 2003.

Taylor, P. J.: Unruly Complexity, Ecology, Interpretation, Engagement, University of Chicago Press, Chicago, 2005.

Ulanowicz, R. E.: Ecology, the ascendent perspective, Columbia University, New York, 1997.

Wardle, D. A., Bardgett, R. D., Klironomos, J. N., Setala, H., van der Putten, W. H., and Wall, D. H.: Ecological linkages between aboveground and belowground biota, Science, 304, 1629-1633, 2004.

Wiens, J. A. and Milne, B. T.: Scaling of 'landscapes' in landscape ecology, or, landscape from a beetle's perspective, Landscape Ecol., 3, 87-96, 1989.

Yarrow, M. M. and Salthe, S. N.: Ecological boundaries in the context of hierarchy theory, Biosystems, 92, 233-244, 2008. 